\title{
DOENÇA CEREBROVASCULAR TIPO "MOYAMOYA"
}

\author{
REynaldo A. Brandt * \\ Charles P. Tilbery ** \\ JUAN R. C. RODRIGUEZ *
}

Takeushi (citado por Suzuki e Takaku ${ }^{19}$ ), em 1961, foi o primeiro a descrever uma doença caracterizada por surtos de isquemia cerebral e aspecto angiográfico típico, constituído por oclusão de ambas as artérias carótidas internas, associada a uma rede angiomatosa na base do cérebro 4, 7, 9, 10, 11, 13, 14, 19, 20, 21. Suzuki e Takaku ${ }^{19}$, ao descreverem o aspecto angiográfico da rede capilar angiomatosa cerebral, compararam-na a "uma baforada de fumaça de cigarro no ar", introduzindo o nome "moyamoya".

Apesar de haver cerca de 400 casos descritos até hoje 7, 8, vários aspectos da doença permanecem obscuros, justificando o relato do presente caso.

\section{OBSERVACAO}

D.G.P., sexo masculino, branco, 34 anos, motorista, atendido em nosso consultório em 14-3-1975, referindo que cinco dias antes, enquanto trabalhava, apresentou súbita e intensa cefaléia occipital, irradiada para a região nucal. Não apresentou alterações de consciência e nem outros sintomas, dirigindo-se a um hospital onde foi submetido a punção suboccipital, com o que a cefaléia diminuiu acentuadamente de intensidade. Antecedentes pessoais - Surtos de cefaléia pulsátil desde há dois anos, ocasião em que sofreu traumatismo. craniano sem consequências imediatas aparentes; úlcera gástrica tratada por gastrectomia há vários anos. Os exames clínico e neurológico foram inteiramente normais. Exames laboratoriais Hemograma evidenciou anemia, com $6,8 \mathrm{~g} \%$ de hemoglobina. O líquido cefalorraqueano obtido no dia do episódio agudo, colhido em outro hospital, mostrou-se hemorrágico, com 25.600 hemácias, 3 leucócitos, $11 \mathrm{mg} \%$ de proteínas, $53 \mathrm{mg} \%$ de glicose, $690 \mathrm{mg} \%$ de cloretos. Punção suboccipital realizada em 14-3-1975 revelou pressão inicial e final de zero, obtendo-se líquido xantocrômico e com indice de cor 8.0; havia 4.869 hemácias degeneradas, 16 leucócitos (52\% linfócitos, 14\% eosinófilos, $10 \%$ monócitos e $24 \%$ reticulócitos, além de macrófagos com hemossiderina), $40 \mathrm{mg} \%$ de proteinas, $77 \mathrm{mg} \%$ de glicose, $735 \mathrm{mg} \%$ de cloretos, $37 \mathrm{mg} \%$ de uréia; reação de Takata-Ara vermelho, reações de Wasserman, VDRL e Weimberg negativas. Eletrencefalograma, em 14-3-1975, mostrou atividade elétrica cerebral de repouso algo irregular, simétrica, por ondas de 9-10 $\mathrm{Hz}$. e $50 \mathrm{microvolts,} \mathrm{predominando}$ em áreas parieto-occipitais. Radiografias de cranio normais. A carotidoangiografia direita (Figs. 1 e 2), realizada em 21-3-1975, mostrou hipoplasia da artéria carótida interna; a artéria oftálmica tem dupla origem, dirigindo-se para a cavidade orbitária. Do segmento intracraniano da carótida interna, nascem várias artérias de pequeno calibre e trajeto tortuoso; destas, predomina uma que nasce logo acima da oftálmica, dirigindo-se superior e anteriormente, e outra que se origina ao nivel

* Neurocirurgiāo; ** Neurologista. 
da emergência da comunicante posterior, na face ântero-lateral da carótida interna, dirigindo-se superior e anteriormente. Ao nivel da origem da artéria corióidea anterior, notam-se várias arteríolas de trajeto tortuoso e ascendente. o conjunto destes vasos tem o aspecto de uma rede vascular anormal, projetando-se na base do cérebro. A artéria comunicante posterior é bem desenvolvida, sendo alongada e projetando-se superior e posteriormente, ultrapassando o nivel do segmento inicial da corióidea anterior e dando origem a arteriolas basais; logo acima da origem da artéria corióidea anterior há estenose da artéria carótida interna, que se afila bruscamente, e acima desta estenose há tênue imagem vascular arredondada. A partir da rede vascular anormal descrita, há tênue contrastação das artérias cerebrais anterior e média. A artéria cerebral posterior é bastante calibrosa e tortuosa. As artérias temporal superficial, occipital e meníngea média são também calibrosas, havendo anastomoses meningo-corticais para o território da artéria cerebral média. A carotidoangiografia esquerda mostrou as artérias carótidas comum e interna hipertrofiadas, com o dobro do diâmetro das correspondentes contralaterais, havendo contrastação dos vasos de ambos os hemisférios cerebrais a partir das mesmas. A artéria comunicante anterior é dupla e calibrosa (tipo fetal), havendo, além das duas artérias cerebrais anteriores, uma terceira, correspondente à artéria mediana do corpo caloso. As artérias lentículo-estriadas contrastam-se bilateralmente, tendo aspecto normal; não há evidência de anastomose entre estas e a rede vascular anormal já descrita. A angiografia vértebro-basilar foi normal. Novo estudo angio-
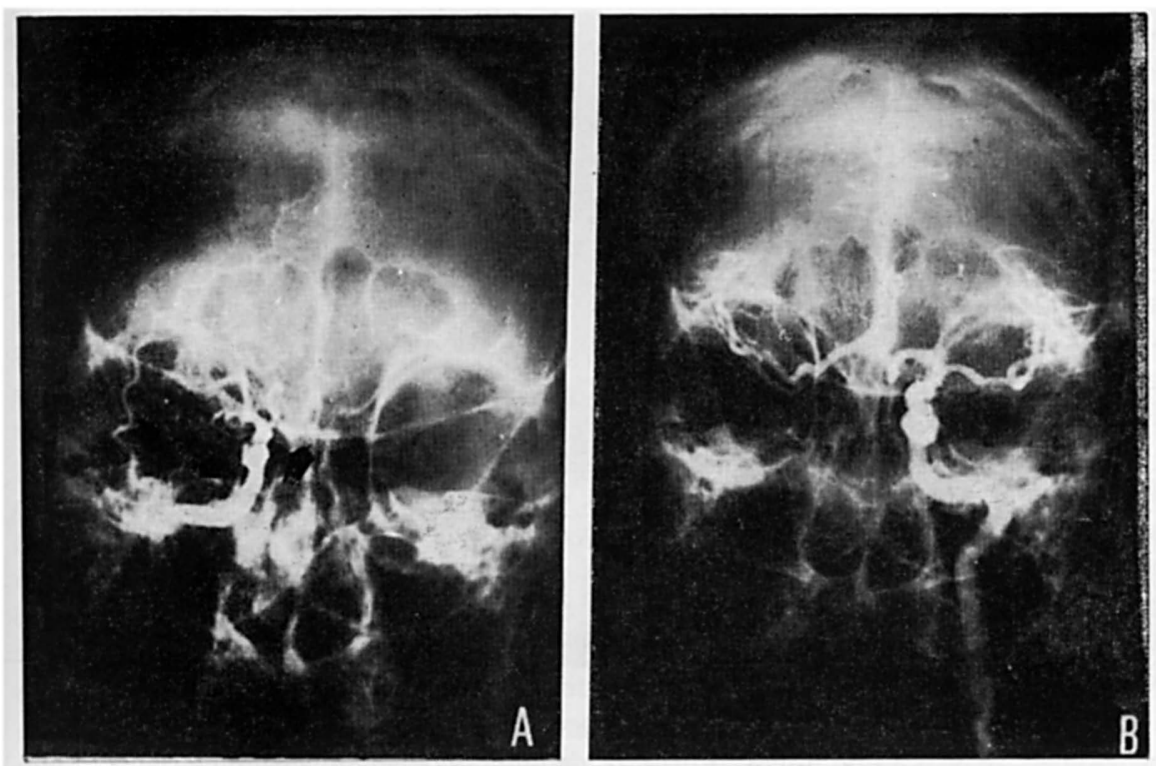

Fig. 1 - Caso D.G.P. Em A, carotidoangiografia direita em projecão ântero-posterior, mostrando oclusão da artéria carótida interna no segmento supraclinóideo; há contrastação de uma rede capilar projetando-se na base do cérebro (setas). Em B, carotidoangiografia esquerda em projeção antero-posterior, mostrando hipertrofia da artéria carótida interna, a partir da qual se contrastam as artérias cerebrais anteriores e médias de ambos os hemisférios cerebrais. A artéría comunicante anterior é calibrosa e entre ambas as artérias cerebrais anteriores se contrasta a artéria mediana no corpo caloso. 

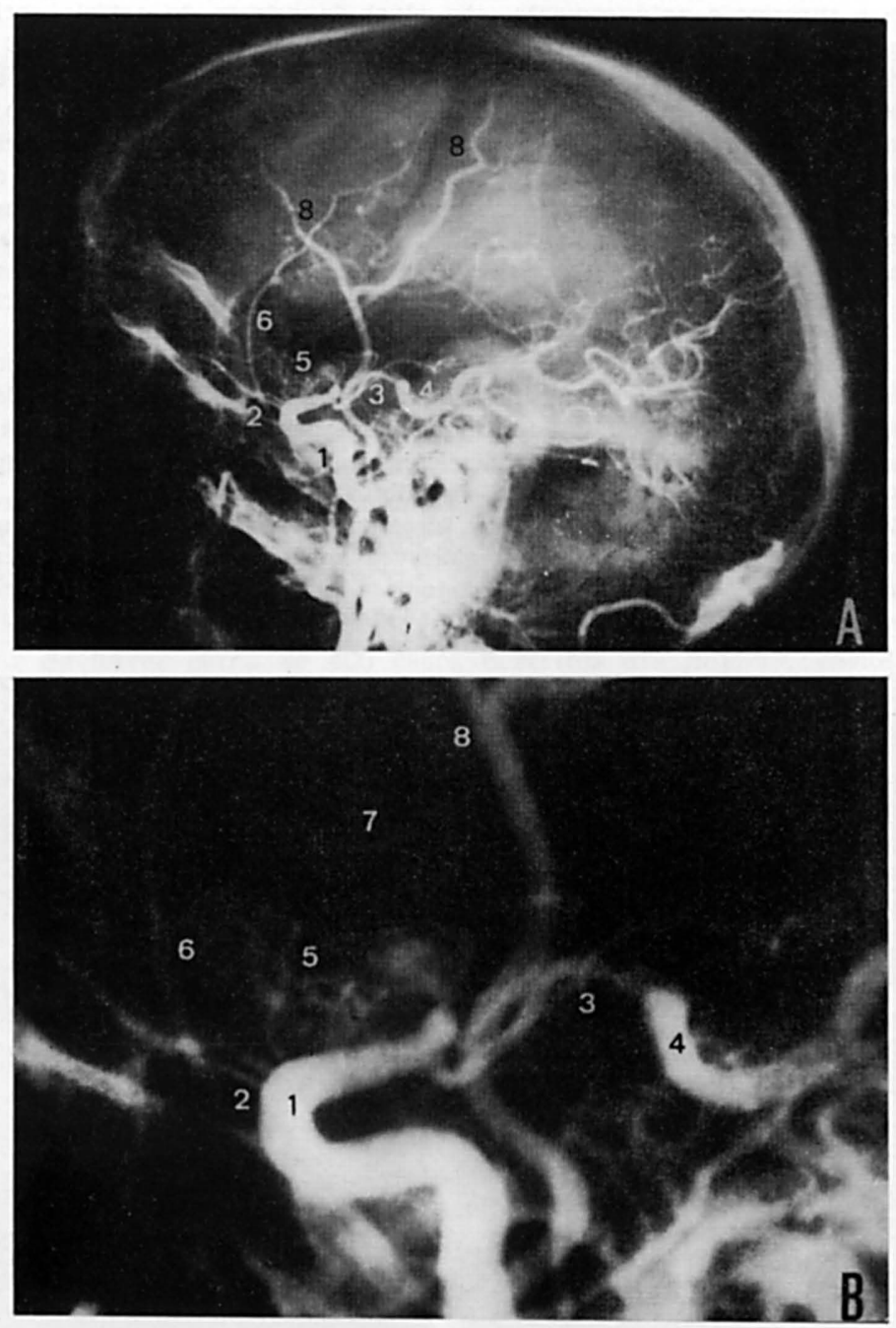

Fig. 2 - Caso D.G.P. Em A, carotidoangiografia direita em projeção lateral e em $B$ detalhe ampliado da mesma, mostrando estenose da artéria carótida interna (1), no segmento supraclinóideo; a artéria oftálmica (2) tem dupla origem, a artéria comunicante posterior (3) é tortuosa e se continua com a artéria cerebral posterior (4) que é calibrosa; há uma rede angiomatosa anormal (5) projetando-se na base do cérebro, a partir da qual há tênue contrastaça das artérias cerebrais anterior (6) e média (7). As artérias meníngeas (8) são calibrosas. 
gráfico realizado seis semanas após o primeiro mostrou as mesmas alteraçōes, sem evidência de progressão das anormalidades descritas.

o paciente evoluiu bem até a presente data, estando assintomático e tendo retornado ao seu trabalho, decorridos 7 meses do episódio hemorrágico.

\section{COMENTARIOS}

A doença de "moyamoya" tem recebido várias outras denominações: doença de Nishimoto-Tekeushi-Kudo ${ }^{22}$, oclusão do círculo de Willis ${ }^{9}$, rede arterial cerebral ${ }^{6}$, telangiectasia cerebral juxtabasal (Sano, citado por Balbo e col. ${ }^{1}$ ), "rete mirabile" basal cerebral ${ }^{13}$, oclusão arterial cerebral progressiva ${ }^{21}$. As primeiras publicações foram de autores japoneses $9,13,14,20,23$, que a consideravam exclusiva da raça amarela. Apesar de terem sido relatados casos no Ocidente ${ }^{1,4}, 7,8,17,21$, há nítido predominio dos mesmos em crianças e adolescentes japoneses do sexo feminino ${ }^{9,14}$.

As manifestações clínicas são de dois tipos principais: crises isquêmicas cerebrais, mais comuns na criança e no adolescente, e hemorragia meníngea, predominante no adulto $4,5,13,14,21$. As crises isquêmicas manifestam-se mais comumente por paresias ${ }^{13},{ }^{14}$, alterações sensitivas ${ }^{5}, 13,14$, convulsões $^{3}$ e co$\mathrm{ma}^{8}$, em episódios frequentemente recorrentes ${ }^{13}, 14$. Esta diferença nas manifestações clínicas levou Kudo ${ }^{9}$ a dividir a doença em duas formas: a juvenil e a do adulto. Na primeira, a doença costuma ser progressiva e, na segunda, estacionária; o prognóstico é bom na maioria dos casos, particularmente nos adultos 9,13 .

$O$ aspecto radiológico é a característica fundamental da doença ${ }^{1,4}, 7,10$, $11,14,17,19,20,21$. Há oclusão ou estenose em graus variáveis da artéria carótida interna, geralmente da sua porção supraclinóidea, com a presença de uma rede vascular angiomatosa na base do cérebro, cujo aspecto é comparado à fumaça de cigarro no ar ("moyamoya"). Na maioria dos casos estas alteraçōes são bilaterais, aparecendo simultaneamente ou em fases sucessivas próximas entre si $^{9,21}$. Quando unilaterais, podem ocorrer outras anormalidades vasculares no lado oposto ao da alteração típica. A rede capilar angiomatosa é visível na fase arterial precoce da angiografia e as artérias cerebrais são geralmente nutridas a partir da mesma. Na maioria dos casos a artéria cerebral posterior é calibrosa, sendo que em muitos a circulação colateral é feita a partir desta e da artéria oftálmica 19. São poucos os casos descritos em que há comprometimento das artérias cerebrais anterior e média ${ }^{16}$, sendo que encontramos apenas um caso descrito de comprometimento associado da artéria basilar ${ }^{8}$. Geralmente a rede capilar angiomatosa é observada ao nivel da base do cérebro, mas há casos descritos de redes capilares corticais ${ }^{7}$, etmoidárias ${ }^{6,20}$, meníngeas e orbitárias ${ }^{6}$. Taveras ${ }^{21}$ chama a atenção para o fato de não haver comprometimento dos ramos distais das artérias cerebrais, o que permite fluxo cortical adequado, desde que haja boa circulação colateral. Esta se faz por três vias principais: 1) anastomoses terminais dos ramos das artérias cerebrais; 2) dilatação das artérias dos núcleos da base, que em alguns casos é capaz de suprir todos os ramos das artérias cerebrais anterior e média; 3) anastomoses transdu- 
rais por ramos das artérias carótidas externa e interna ("rete mirabile"). Em nosso caso, a circulação colateral para as artérias cerebrais anterior e média se fez à custa da artéria carótida interna contralateral, além da que se fez a partir da rede capilar angiomatosa e das anastomoses transdurais.

Segundo Taveras ${ }^{21}$, a doença oclusiva é progressiva, sendo que Suzuki e Takaku ${ }^{19}$ distinguem 6 fases de evolução da mesma: 1) estreitamento do sifão carotídeo; 2) aparecimento do "moyamoya" basal; 3) desenvolvimento acentuado do "moyamoya" basal; 4) redução do "moyamoya" basal com evolução da estenose carotídea e suplementação da circulação cerebral através de ramos das artérias carótida externa e oftálmica; 5) obstrução da carótida interna que atinge o segmento $\mathrm{C} 2$, com desaparecimento dos segmentos iniciais das artérias cerebrais anterior e média e redução acentuada do "moyamoya" basal, com aumento da circulação colateral a partir da artéria carótida externa; 6) desaparecimento do "moyamoya" basal e da porção supracavernosa da artéria carótida interna, dependendo a circulação cerebral dos ramos das artérias carótidas externas. Alguns autores 4, 13, 14, 21 indicam estudo angiográficos dos 4 troncos arteriais cerebrais, enquanto que Iivanainem e col. ${ }^{7}$ contraindicam o estudo bilateral, temendo complicações decorrentes do mesmo.

As descrições anátomo-patológicas da doença são raras 14, 16, 18, 23. Nestas, o polígono de Willis é hipoplásico, com a luz vascular diminuída ou ocluída ao nível do sifão carotídeo, logo acima da origem da artéria comunicante posterior. No caso descrito por Perret e col. ${ }^{16}$, o segmento estenosado da artéria carótida interna estava reduziđo a um cordão fibroso, com aspecto de atresia. A histopatologia revela hipoplasia dos vasos, com espessamento da íntima e desdobramento da limitante elástica interna; por vezes há edema e entumescimento mucóide das paredes vasculares, que podem levar à obstrução completa da luz arterial. Nas bifurcações arteriais, os coxins fibroblásticos apresentam-se hipertróficos, podendo a membrana elástica interna ser, ou não, bem desenvolvida. Na túnica média há alterações segmentares, irregulares, por vezes com fibrose e atrofia. Nos estudos feitos não foram encontrados sinais inflamatórios e nem de ateromasia 14, 16, 23.

A etiologia da doença não é conhecida. Há casos descritos com antecedentes de doenças inflamatórias da cabeça e pescoço, não havendo entretanto correlação cronológica direta destas com o aparecimento de sinais e sintomas neurológicos ${ }^{14,19}$. Alguns autores $6,9,20,21$ sugerem que a oclusão da artéria carótida interna seria consequência de um processo inflamatório crônico; o "moyamoya" representaria um tipo de suplência colateral, a partir de artérias perfurantes, consequente à estenose da artéria carótida interna. Outros $3,5,12,14,18$ sugerem que a doença constitui malformação vascular, com persistência de uma rede vascular embrionária. Alguns fatos apoiam esta teoria: há predomínio racial da doença $9,14,19,21$, há casos com antecedentes familiares de moléstias vasculares, as lesões são frequentemente bilaterais e simétricas, há hipoplasia e por vezes atresia das artérias carótidas internas, há persistência de artérias embrionárias como a artéria an- 
terior da foice do cérebro e de anastomoses carótido-basilares, além de associação com aneurismas intracranianos ${ }^{5}, 10,12$; as artérias cerebrais anterior e média não se originam diretamente da artéria carótida interna, mas de uma rede vascular anormal, de aspecto embrionário ${ }^{13,14}$, formando uma "rete mirabile" de vasos livremente anastomosados nas proximidades do polígono de Willis. Vuia e col. ${ }^{23}$ acreditam que as alterações do "moyamoya" sejam o resultado de uma afecção congênita, à qual se associa um processo evolutivo. As artérias hipoplásicas do poligono de Willis estariam sujeitas a uma tensão arterial comparável à sofrida por vasos normais na hipertensão arterial; consequentemente, haveria proliferação crônica da endartéria, facilitando a trombose que, por sua vez, agravaria a insuficiência circulatória cerebral. A compensação hemodinâmica se explicaria pela evolução lenta e progressiva da oclusão vascular, pela persistência de anastomoses através da rede capilar angiomatosa e pelo desenvolvimento de vias de circulação colateral 6, 10, 11, 23. Segundo Perret e col. 16, a estenose das artérias carótidas e a formação da rede vascular tipo "moyamoya" far-se-iam em tempos diferentes da vida embrionária, fetal e pós-natal. Handa e Handa ${ }^{6}$ acreditam que processos infecciosos, antecedendo a instalação do quadro neurológico, precipitariam uma descompensação funcional do território vascular estenosado. De modo semelhante, as manifestações do adulto podem ser devidas à diminuição das reservas funcionais da circulação cerebral ${ }^{5}$.

No presente caso, a doença manifestou-se em adulto branco, com hemorragia meningea, sem sinais de comprometimento cerebral. O estudo angiográfico revelou as alterações características do "moyamoya", havendo hipoplasia da artéria carótida interna direita, com estenose do seu segmento supraclinóideo, acima da emergência da artéria comunicante posterior. Do segmento pré-estenótico, acima da origem dupla da artéria oftálmica, nascem múltiplas arteriolas, das quais duas se destacam: uma, que se origina na direção oposta à da artéria comunicante posterior e, outra, na direção oposta à da artéria corióidea anterior. De acordo com as descrições de Padget ${ }^{15}$, estas duas arteríolas parecem corresponder aos remanescentes das artérias oftálmicas primitivas, dorsal e ventral. Juntamente com as demais, formam uma rede vascular anormal, que se projeta na região basal do cérebro, semelhante à rede vascular basal do embrião ${ }^{2}$. A partir desta rede capilar e das anastomoses meningo-corticais, há tênue contrastação das artérias cerebrais anterior e média, constituindo uma circulação precária e insuficiente. A suplência sanguínea do hemisfério cerebral direito provém prin cipalmente da artéria carótida interna esquerda, hipertrofiada e vicariante, através do polígono de Willis; neste, a artéria comunicante anterior é dupla e hipertrofiada, de tipo fetal. Nota-se ainda a presença anômala da artéria mediana do corpo caloso entre as duas artérias cerebrais anteriores. As artérias comunicante posterior e cerebral posterior direitas continuam-se diretamente uma com a outra, tendo a artéria comunicante posterior um trajeto curvilíneo e atípico, ultrapassando o nível da artéria corióidea anterior, lembrando a curvatura embrionária descrita por Padget ${ }^{15}$ no embrião de 5,3 a $9 \mathrm{~mm}$. 
Não encontramos outro caso descrito em que coexistissem as anormalidades típicas do "moyamoya" de um lado com hipertrofia da artéria carótida interna do lado oposto. Estas alterações, ao lado do aspecto fetal da artéria comunicante anterior, da persistência da artéria mediana do corpo caloso e do percurso anômalo da artéria comunicante posterior, além da rede vascular angiomatosa anormal na base do cérebro, são, para nós, sinais sugestivos de uma anomalia do desenvolvimento. É possível que fatores adquiridos contribuam para o desencadeamento de sinais e sintomas neurológicos; não nos parece, no entanto, serem estes os únicos responsáveis pelas alterações angiográficas pois, ao menos no presente caso, o desenvolvimento de uma rede capilar na base do cérebro seria desnecessário para assegurar a circulação colateral para o hemisfério cerebral direito. Esta rede capilar parece fazer parte de uma malformação vascular mais ampla, com as carcterísticas do "moyamoya", constituindo uma entidade patológica definida.

\section{RESUMO}

E relatado o caso de um paciente masculino, branco, 34 anos, que apresentou hemorragia meningea sem sinais de comprometimento cerebral. A carotidoangiografia direita mostrou as alterações típicas do "moyamoya", com estenose da artéria carótida interna ao nível do sifão, acima da origem da artéria comunicante posterior; há uma rede vascular angiomatosa anormal projetando-se na base do cérebro, da qual nascem as artérias cerebrais anterior e média, sendo as artérias comunicante e cerebral posterior hipertrofiadas e de trajeto tortuoso; há anastomoses meningo-corticiais, sendo as artérias meníngeas hipertrofiadas. A carotidoangiografia esquerda mostrou hipertrofia das artérias carótidas comum e interna, com contrastação de ambas as artérias cerebrais anteriores e médias a partir deste lado; a artéria comunicante anterior é do tipo fetal e há a presença anômala da artéria cerebral anterior mediana do corpo caloso. Estes dados apoiam a teoria segundo a qual a doença constitui anomalia do desenvolvimento vascular encefálico, não tendo sido encontrado outro caso na literatura com hipertrofia vicariante da artéria carótida interna contralateral à da lesão típica do "moyamoya".

\section{SUMMARY}

\section{Cerebrovascular "moyamoya" disease: a case report and review of the literature}

The case of a 34-year-old caucasian male with subarachnoid hemorrahge is reported. The right carotid arteriogram shows the typical internal carotid artery occlusion at the syphon, with an abnormal vascular network at the base of the brain; the right anterior and middle cerebral arteries fill from the vascular network and through meningocortical anastomoses; the right posterior communicating and cerebral arteries are tortuous and 
hypertrophic. The left carotid arteriogram shows hypertrophy of the common and internal carotid arteries; both anterior and middle cerebral arteries fill from this side; the anterior communicating artery is thick (foetal type) and a median anterior cerebral artery to the corpus callosum is present. It is the authors' view that the "moyamoya" disease is a congenital one, since the abnormal vascular network at the base of the brain would not be necessary, in this case, to preserve good collateral circulation to the right cerebral hemisphere. No other case with the typical findings of "moyamoya" disease on one side and hypertrophy of the carotid arteries on the other was found in the literature.

\section{REFERENCIAS}

1. BALBO, R. J.; SARIAN, L.; SPERLECU, A. \& RIBEIRO, L. A. O. - Doença oclusiva progressiva das artérias cerebrais (moyamoia). Seara Med. Neuroc. (S. Paulo) 2:166, 1972 .

2. BREMER, J. L. - Congenital aneurysms of the cerebral arteries. Arch. Path. (Chicago) 35:819, 1943.

3. DAMME van, W.; BEECKMAN, P. \& VERBRUGGEN, R. - Moyamoya syndrome probably associated with hydrocephalus. Neuroradiology 9:39, 1975 .

4. DILLON, J. D.; STOKES, H. \& MEIROWSKY, A. M. - Moyamoya disease. Surg. Neurol. 3:233, 1975.

5. GALligiONI, F.; ANDRIOLT, G. C.; MARIN, G.; BRIANI, S. \& IRACl, G. Hypoplasia of the internal carotid artery associated with cerebral pseudoangiomatosis. Amer. J. Roentgenol. 112:251, 1971.

6. HANDA, J. \& HANDA, H. - Progressive cerebral arterial occlusive disease: analysis of 27 cases. Neuroradiology $3: 119,1972$.

7. IIVANAINEN, M.; VUOLIO, M. \& HALONEN, V. - Occlusive disease of intracranial main arteries with collateral networks (moyamoya disease) in adults. Acta Neurol. Scandinav. 49:307, 1973.

8. KRAYENBÜHL, H. A. - The moyamoya syndrome and the neurosurgeon. Surg. Neurol. 4:353, 1975 .

9. KUDO, T. - Spontaneous occlusion of the circle of Willis. A disease apparently confined to japanese. Neurology (Minneapolis) 18:485, 1968.

10. LEE, M. L. K. \& CHEUNG, E. M. T. - Moyamoya disease as a cause of subarachnoid haemorrhage in chinese. Brain 96:623, 1973.

11. LEEDS, N. E. \& ABBOTT, K. H. - Collateral circulation in cerebrovascular disease in childhood via rete mirabile and perforating branches of anterior choroidal and posterior cerebral arteries. Radiology 85:628, 1965.

12. LEPOIRE, J.; TRIDON, P.; MONTAUT, J.; HEPNER, J.; RENARD, M. \& PICARD, L. - Malformations angiomateuses artério-artérielles du système carotidien. Neurochirurgie 15:5, 1969.

13. NISHIMOTO, A. \& TAKEUSHI, S. - Abnormal cerebrovascular network related to the internal carotid arteries. J. Neurosurg. 29:255, 1968.

14. NISHIMOTO, A. \& TAKEUSHI, S. - Moyamoya disease. Abnormal cerebrovascular network in the cerebral basal region. In VINKEN, P. J. \& BRUYN, G. W. - Handbook of Clinical Neurology, vol. 12, North-Holland, Amsterdan, 1972 , p. 352 .

15. PADGET, D. H. - The circle of Willis. Its embriology and anatomy. In DANDY, W. - Intracranial arterial aneurysms. Comstock, 1944, p. 67.

16. PERRET, J.; CROUZET, G.; PELLAT, J.; PASQUIER, B.; LARRIBAU, E. \& CHIROSSEL, J. P. - Sténose sylvienne avec réseaux de type moya moya. Rev. Neurol. (Paris) 131:243, 1975.

17. PRENSKY, A. L. \& DAVIS, D. O. - Obstruction of major cerebral vessels in early childhood without neurological signs. Neurology (Minneapolis) 20:945, 1970 . 
18. SMITH, K. R.; NELSON, J. S. \& DOOLEY, J. M. - Bilateral "hypoplasia" of the internal carotid arteries. Neurology (Minneapolis) 18:1149, 1968.

19. SUZUKI, J. \& TAKAKU, A. - Cerebrovascular "moyamoya" disease. Arch. Neurol. (Chicago) 20:288, 1969.

20. SUZUKI, J. \& KODAMA, N. - Cerebrovascular "moyamoya" disease. Angiology 22:223, 1971.

21. TAVERAS, J. M. - Multiple progressive intracranial arterial occlusions: a syndrome of children and young adults. Amer. J. Roentgenol. 106:235, 1969.

22. URBANEK, K.; FARKOVA, H. \& KLAUS, E. - Nishimoto-Takeushi-Kudo disease: case report. J. Neurol. Neurosurg. Psychiat. 33:671, 1970.

23. VUIA, O.; ALEXIANU, M. \& GABOR, S. - Hypoplasia and obstruction of the circle of Willis in a case of atypical cerebral hemorrhage and its relationship to Nishimoto's disease. Neurology (Minneapolis) 20:361, 1970.

Unidade Neurológica e Neurocirürgica - Praça Oswaldo Cruz 138, cj. 73/74 - 04004 São Paulo, SP - Brasil. 